\title{
Lattice points in a circle: An improved mean-square asymptotics
}

by

\author{
Werner Georg NowaK (Wien)
}

\section{Introduction. Let as usual}

$$
r(n):=\#\left\{(u, v) \in \mathbb{Z}^{2}: u^{2}+v^{2}=n\right\}
$$

denote the number of ways to write the integer $n$ as a sum of two squares. Then the classic circle problem, which goes back to C. F. Gauß, is concerned with the asymptotic behaviour of the quantity

$$
P(x):=\sum_{0 \leq n \leq x} r(n)-\pi x .
$$

In other words, this is the lattice point discrepancy of a compact, origincentered circular disc with radius $\sqrt{x}$. For detailed and enlightening expositions of the rich history of this topic, the reader should consult the monographs of E. Krätzel [10], [11].

The sharpest upper bound has been established quite recently by M. Huxley [7] (as a slight improvement on Huxley [6]) and reads

$$
P(x) \ll x^{131 / 416}(\log x)^{18637 / 8320},
$$

where $131 / 416=0.3149 \ldots$ Concerning lower bounds, significant progress has just been achieved by K. Soundararajan [20] who proved that

$$
P(x) \neq o\left(x^{1 / 4}(\log x)^{1 / 4}(\log \log x)^{3\left(2^{1 / 3}-1\right) / 4}(\log \log \log x)^{-5 / 8}\right) .
$$

For a longer time, it has been known that

$$
\liminf _{x \rightarrow \infty}\left(\frac{P(x)}{x^{1 / 4}(\log x)^{1 / 4} \omega_{1}(x)}\right)<0, \quad \limsup _{x \rightarrow \infty}\left(\frac{P(x)}{x^{1 / 4} \omega_{2}(x)}\right)>0,
$$

with

$$
\begin{aligned}
& \omega_{1}(x):=(\log \log x)^{(\log 2) / 4} \exp \left(-c_{1}(\log \log \log x)^{1 / 2}\right), \\
& \omega_{2}(x):=\exp \left(c_{2}(\log \log x)^{1 / 4}(\log \log \log x)^{-3 / 4}\right),
\end{aligned}
$$

2000 Mathematics Subject Classification: 11P21, 11N37, 11N25, 11N36. 
$c_{1}, c_{2}$ appropriate positive constants. These results are due to J. L. Hafner [4], resp. K. Corrádi and I. Kátai [2].

It is usually conjectured that $x^{1 / 4}$, as it appears in (1.4), essentially meets the "true" order of $P(x)$, i.e., that

$$
\inf \left\{\lambda: P(x) \ll_{\lambda} x^{\lambda}\right\}=1 / 4 .
$$

In favour of this hypothesis, there are quite precise mean-square asymptotics of the shape

$$
\int_{0}^{X}(P(x))^{2} d x=C X^{3 / 2}+Q(X),
$$

with

$$
\begin{aligned}
C & =\frac{1}{3 \pi^{2}} \sum_{n=1}^{\infty} r^{2}(n) n^{-3 / 2}=\frac{16}{3 \pi^{2}} \frac{\zeta^{2}(3 / 2) L^{2}(3 / 2)}{\zeta(3)}\left(1+2^{-3 / 2}\right)^{-1} \\
& \approx 1.69396 .
\end{aligned}
$$

Here and throughout, $L(s)$ denotes the $L$-series corresponding to the nonprincipal character modulo 4.

The estimation of the remainder $Q(X)$ has been subject of intensive research, by increasingly sophisticated methods. We mention the results of H. Cramér [3]: $Q(X) \ll X^{5 / 4+\varepsilon}$, E. Landau [12]: $Q(X) \ll X^{1+\varepsilon}$, and A. Walfisz [21]: $Q(X) \ll X(\log X)^{3}$. The sharpest bound to date is due to I. Kátai [9] and reads

$$
Q(X) \ll X(\log X)^{2}
$$

More recently, E. Preissmann [17] found a short and elegant proof for this result, using a deep inequality of Montgomery and Vaughan. (See Lemma B below.)

The objective of the present note is to obtain a further reduction of the log-exponent in the estimate (1.8).

THEOREM. The error term $Q(X)$ defined by (1.6), (1.7) satisfies, as $X \rightarrow \infty$,

$$
Q(X) \ll X(\log X)^{3 / 2} \log \log X
$$

Remarks. 1. Compared to the previous authors cited, we simplify the analysis by an approach suggested by T. Meurman for the divisor problem [14]. The basic idea of our refinement is that most integers are not representable as a sum of two squares, and that the so-called $\mathfrak{B}$-numbers (i.e., those with $r(n)>0$ ) are usually well-spaced-i.e., "B-twins" are still less frequent. This fact permits a small extra saving when applying the Montgomery-Vaughan bound with care. 
2. It may be instructive to report the present state-of-the-art with the analogous error term $Q_{\Delta}(X)$ corresponding to the Dirichlet divisor problem. According to E. Preissmann [17], it is known that $Q_{\Delta}(X) \ll X(\log X)^{4}$, while Y.-K. Lau and K.-M. Tsang [13] recently proved that $Q_{\Delta}(X)$ is not a $o\left(X(\log X)^{2}\right)$. This might suggest that our result is not far away from the best possible bound.

\section{Some auxiliary results}

NotATion. Variables of summation automatically range over all integers satisfying the conditions indicated. $p$ denotes primes throughout, and $\mathbb{P}$ is the set of all (rational) primes. For any subset $\mathfrak{P} \subseteq \mathbb{P}$, we write $\mathfrak{D}(\mathfrak{P})$ for the set of all positive integers $m$ whose prime divisors are all in $\mathfrak{P}$. All constants implied in the symbols $O(\cdot), \ll, \gg$, etc., are absolute, and $\varepsilon$ denotes a sufficiently small positive constant.

Lemma A. Suppose that $x \geq 1, x \notin \mathbb{Z}$, and $x \leq M \leq x^{A}$, where $A>1$ is some fixed constant. Denote by $\|\cdot\|$ the distance from the nearest integer. Then, for every $\varepsilon>0$,

$$
\begin{aligned}
P(x)= & \sqrt{x} \sum_{1 \leq n<M} r(n) n^{-1 / 2} J_{1}(2 \pi \sqrt{n x}) \\
& +O\left(\min \left(x^{5 / 4} M^{-1 / 2}+x^{1 / 2+\varepsilon} M^{-1 / 2}\|x\|^{-1}+x^{1 / 4} M^{-1 / 4}, x^{\varepsilon}\right)\right)
\end{aligned}
$$

where $J_{1}$ is a Bessel function.

Proof. This is Lemma 1 in A. Ivić [8], combined with his eq. (1.7).

Lemma B. For an arbitrary finite index set $\mathcal{J}$, let $\left(a_{j}\right)_{j \in \mathcal{J}}$ be a complex sequence and let $\left(\lambda_{j}\right)_{j \in \mathcal{J}}$ be a sequence of pairwise distinct reals. Write

$$
\delta_{j}:=\min _{k \in \mathcal{J}, k \neq j}\left|\lambda_{k}-\lambda_{j}\right| .
$$

Then, for arbitrary real $T_{0}$ and $T>0$,

$$
\int_{T_{0}}^{T_{0}+T}\left|\sum_{j \in \mathcal{J}} a_{j} \exp \left(i \lambda_{j} t\right)\right|^{2} d t=T \sum_{j \in \mathcal{J}}\left|a_{j}\right|^{2}+O\left(\sum_{j \in \mathcal{J}} \frac{\left|a_{j}\right|^{2}}{\delta_{j}}\right),
$$

where the O-constant is absolute.

Proof. This is an obvious variant of Corollary 2 in H. L. Montgomery and R. C. Vaughan [15].

Lemma C. For each prime power $p^{\alpha}, \alpha \geq 1$, let $\bar{\Omega}\left(p^{\alpha}\right)$ be a set of distinct residue classes $\overline{\mathbf{c}}$ modulo $p^{\alpha}$. Define further

$$
\Omega\left(p^{\alpha}\right)=\left\{n \in \mathbb{Z}_{+}: n \in \bigcup_{\overline{\mathfrak{c}} \in \bar{\Omega}\left(p^{\alpha}\right)} \overline{\mathfrak{c}}\right\}, \quad \theta\left(p^{\alpha}\right)=1-\sum_{j=1}^{\alpha} \frac{\# \bar{\Omega}\left(p^{j}\right)}{p^{j}}, \quad \theta(1)=1 .
$$


Suppose that $\Omega\left(p^{\alpha}\right) \cap \Omega\left(p^{\beta}\right)=\emptyset$ for all primes $p$ and positive integers $\alpha \neq \beta$. For real $x>0$, let finally

$$
A(x)=\left\{n \in \mathbb{Z}_{+}: n \leq x \text { and } n \notin \bigcup_{p \in \mathbb{P}, \alpha \in \mathbb{Z}_{+}} \Omega\left(p^{\alpha}\right)\right\} .
$$

Then, for arbitrary real $D>1$,

$$
\# A(x) \leq \frac{x+D^{2}}{V_{D}}, \quad \text { where } \quad V_{D}:=\sum_{0<d<D} \prod_{p^{\alpha} \| d}\left(\frac{1}{\theta\left(p^{\alpha}\right)}-\frac{1}{\theta\left(p^{\alpha-1}\right)}\right) .
$$

Proof. This is a deep sieve theorem due to A. Selberg [19]. It can be found in Y. Motohashi [16, p. 11, Theorem 2], and also in T. Cochrane and R. E. Dressler [1].

LeMma D. Let $\left(a_{n}\right)_{n \in \mathbb{Z}_{+}}$be a sequence of nonnegative reals, and suppose that the Dirichlet series

$$
f(s)=\sum_{n=1}^{\infty} a_{n} n^{-s}
$$

converges for $\Re(s)>1$. Assume further that, for some real constants $A$ and $\gamma>0$

$$
f(s)=(A+o(1))(s-1)^{-\gamma}
$$

as $s \rightarrow 1+$. Then, as $x \rightarrow \infty$,

$$
\sum_{1 \leq n \leq x} \frac{a_{n}}{n}=\left(\frac{A}{\Gamma(1+\gamma)}+o(1)\right)(\log x)^{\gamma} .
$$

Proof. This is a standard Tauberian theorem. For the present formulation, cf. T. Cochrane and R. E. Dressler [1, Lemma B].

Lemma E. Let as usual

$$
\mathfrak{B}=\{n \in \mathbb{Z}: r(n)>0\},
$$

and let $\mathbf{b}: \mathbb{Z} \rightarrow\{0,1\}$ denote the indicator function of $\mathfrak{B}$. Then, for each integer $n>0$,

$$
r^{2}(n) \leq \sum_{\substack{k m=n \\ k, m>0}} r(k) r(m)
$$

and

$$
r(n) \leq 4 \sum_{\substack{k m=n \\ k, m>0}} \mathbf{b}(k) \mathbf{b}(m)
$$


Proof. Recall the explicit formula for $r(n)$ (cf., e.g., [5, p. 60]): $\frac{1}{4} r(n)$ (and hence also $\mathbf{b}(n)$ ) is multiplicative; for $p$ prime and any integer $k \geq 0$,

$$
\frac{1}{4} r\left(p^{k}\right)= \begin{cases}k+1 & \text { if } p \equiv 1 \bmod 4 \\ 0 & \text { if } p \equiv 3 \bmod 4 \text { and } k \text { is odd } \\ 1 & \text { if } p \equiv 3 \bmod 4 \text { and } k \text { is even, } \\ 1 & \text { if } p=2 .\end{cases}
$$

By multiplicativity, it suffices to verify (2.1) and (2.2) for prime powers. The only case not completely obvious is that of $(2.1)$ for $p \equiv 1 \bmod 4$, where we have to show that

$$
(k+1)^{2} \leq \sum_{j=0}^{k}(j+1)(k-j+1) .
$$

Since the right hand side equals

$$
\frac{1}{6}(k+1)(k+2)(k+3)=(k+1)^{2}+\frac{1}{6} k(k+1)(k-1),
$$

this is clear as well.

Lemma F. Let $\phi$ denote the Euler totient function. Then, for $y \geq 2$,

$$
\begin{aligned}
\sum_{0<n \leq y}\left(\frac{n}{\phi(n)}\right)^{2} \frac{\mathbf{b}(n)}{n} \ll(\log y)^{1 / 2}, \\
\sum_{0<n \leq y}\left(\frac{n}{\phi(n)}\right)^{2} \frac{r(n)}{n} \ll \log y .
\end{aligned}
$$

Proof. Recall that, for $\Re(s)>1$, the Dedekind zeta-function of the Gaussian field satisfies

$$
\zeta_{\mathbb{Q}(i)}(s)=\sum_{n=1}^{\infty} \frac{1}{4} r(n) n^{-s}=g_{1}(s) \prod_{p \equiv 1 \bmod 4}\left(1-p^{-s}\right)^{-2} ;
$$

here and throughout, $g_{1}(s), g_{2}(s), \ldots$ denote functions which are holomorphic (at least) in the closed half-plane $\Re(s) \geq 1$. Hence, for $\Re(s)>1$,

$$
\begin{aligned}
f_{1}(s):= & \sum_{n=1}^{\infty}\left(\frac{n}{\phi(n)}\right)^{2} \mathbf{b}(n) n^{-s} \\
= & g_{2}(s) \prod_{p \equiv 1 \bmod 4}\left(1+\left(1-\frac{1}{p}\right)^{-2} \sum_{k=1}^{\infty} p^{-k s}\right) \\
= & g_{3}(s)\left(\zeta_{\mathbb{Q}(i)}(s)\right)^{1 / 2} \\
& \times \prod_{p \equiv 1 \bmod 4}\left\{\left(1+\left(1-\frac{1}{p}\right)^{-2} \frac{p^{-s}}{1-p^{-s}}\right)\left(1-p^{-s}\right)\right\} \\
= & g_{3}(s)\left(\zeta_{\mathbb{Q}(i)}(s)\right)^{1 / 2} \prod_{p \equiv 1 \bmod 4}\left(1+\frac{2 p-1}{(p-1)^{2}} p^{-s}\right) .
\end{aligned}
$$


Since the last product converges absolutely for $\Re(s)>0$, it follows that $f_{1}(s)=g_{4}(s)\left(\zeta_{\mathbb{Q}(i)}(s)\right)^{1 / 2}$ in $\Re(s)>1$, hence

$$
f_{1}(s)=\left(g_{4}(1) \frac{\sqrt{\pi}}{2}+o(1)\right)(s-1)^{-1 / 2} \quad \text { as } s \rightarrow 1+.
$$

Thus Lemma D immediately implies (2.4).

To establish (2.5), we similarly consider, for $\Re(s)>1$,

$$
\begin{aligned}
f_{2}(s):= & \sum_{n=1}^{\infty}\left(\frac{n}{\phi(n)}\right)^{2} \frac{1}{4} r(n) n^{-s} \\
= & g_{5}(s) \prod_{p \equiv 1 \bmod 4}\left(1+\left(1-\frac{1}{p}\right)^{-2} \sum_{k=1}^{\infty}(k+1) p^{-k s}\right) \\
= & g_{6}(s) \zeta_{\mathbb{Q}(i)}(s) \\
& \times \prod_{p \equiv 1 \bmod 4}\left\{\left(1+\left(1-\frac{1}{p}\right)^{-2} \frac{p^{-s}\left(2-p^{-s}\right)}{\left(1-p^{-s}\right)^{2}}\right)\left(1-p^{-s}\right)^{2}\right\} .
\end{aligned}
$$

The last product (call it $H(s)$ ) converges absolutely for $s=1$, namely

$$
H(1)=\prod_{p \equiv 1 \bmod 4}\left(1+\frac{(2 p-1)^{2}}{p^{2}(p-1)^{2}}\right) .
$$

Thus

$$
f_{2}(s)=\left(g_{6}(1) H(1) \frac{\pi}{4}+o(1)\right)(s-1)^{-1} \quad \text { as } s \rightarrow 1+,
$$

and one more appeal to Lemma D completes the proof of Lemma F.

\section{Sums over $\mathfrak{B}$-twins}

Proposition 1. For integers $k>0$ and $h \neq 0$, and large real $x$,

$$
S_{0}(k, h ; x):=\sum_{0<n \leq x} \mathbf{b}(n) \mathbf{b}(k n+h) \ll\left(\frac{k|h|}{\phi(k|h|)}\right)^{2} \frac{x}{\log x},
$$

where $\phi$ is the Euler totient function.

Proof. For $k=1$ this is a classic and celebrated result of G. J. Rieger [18]. Instead of working out his argument for the general case, we prefer to follow the approach of T. Cochrane and R. E. Dressler [1] who used a deeper theorem of Selberg's (our Lemma C) to deal with the problem of $\mathfrak{B}$-triples. Let

$$
\mathfrak{P}=\mathfrak{P}_{k, h}=\{p \in \mathbb{P}: p \equiv 3 \bmod 4 \text { and } p \nmid k h\} .
$$


Further, for $p \in \mathfrak{P}, \alpha \in \mathbb{Z}_{+}$, denote by $\overline{k_{p^{\alpha}}^{*}}$ the residue class modulo $p^{\alpha}$ for which $\bar{k} \overline{k_{p^{\alpha}}^{*}}=\overline{1}$. In the notation of Lemma C, we choose

$$
\bar{\Omega}\left(p^{\alpha}\right):=\left\{\overline{j p^{\alpha-1}}: j=1, \ldots, p-1\right\} \cup\left\{\overline{k_{p^{\alpha}}^{*}\left(j p^{\alpha-1}-h\right)}: j=1, \ldots, p-1\right\}
$$

if and only if

$$
p \in \mathfrak{P} \text { and } \alpha \text { is an even positive integer, }
$$

and $\bar{\Omega}\left(p^{\alpha}\right):=\emptyset$ in all other cases. Thus it is easy to see that, in the case $(*), \# \bar{\Omega}\left(p^{\alpha}\right)=2(p-1)$, and

$$
n \in \Omega\left(p^{\alpha}\right) \Rightarrow\left(p^{\alpha-1} \| n \text { or } p^{\alpha-1} \|(k n+h)\right) \Rightarrow \mathbf{b}(n) \mathbf{b}(k n+h)=0 .
$$

Therefore, in the terminology of Lemma C,

$$
S_{0}(k, h ; x) \leq \# A(x) \leq \frac{x+D^{2}}{V_{D}} \leq \frac{2 x}{V_{D}},
$$

if we choose $D:=\sqrt{x}$. To find a lower bound for $V_{D}$, we note that

$$
\theta\left(p^{\alpha}\right)=1-2(p-1) \sum_{0<2 \beta \leq \alpha} p^{-2 \beta} \quad \text { for } p \in \mathfrak{P},
$$

hence

$$
\theta\left(p^{\alpha}\right)= \begin{cases}1-\frac{2}{p+1}+\frac{2}{p^{\alpha}(p+1)} & \text { for } p \in \mathfrak{P} \text { and } \alpha>0 \text { even, } \\ \theta\left(p^{\alpha-1}\right) & \text { for } p \in \mathfrak{P} \text { and } \alpha \text { odd } \\ 1 & \text { if } p \notin \mathfrak{P}\end{cases}
$$

Therefore,

$$
\frac{1}{\theta\left(p^{\alpha}\right)}-\frac{1}{\theta\left(p^{\alpha-1}\right)}=\left\{\begin{array}{l}
\frac{2 p^{\alpha}(p+1)\left(p^{2}-1\right)}{\left(p^{\alpha}(p-1)+2\right)\left(p^{\alpha}(p-1)+2 p^{2}\right)} \\
\text { if } p \in \mathfrak{P} \text { and } \alpha>0 \text { even }, \\
0 \quad \text { in all other cases. }
\end{array}\right.
$$

Consequently, in the sum defining $V_{D}$, we can restrict $d$ to perfect squares, and moreover to the set $\mathfrak{D}(\mathfrak{P})$. Thus

$$
\begin{aligned}
V_{D} & =\sum_{\substack{0<d_{1}<\sqrt{D} \\
d_{1} \in \mathfrak{D}(\mathfrak{P})}} \prod_{p^{\beta} \| d_{1}}\left(\frac{1}{\theta\left(p^{2 \beta}\right)}-\frac{1}{\theta\left(p^{2 \beta-2}\right)}\right) \\
& \geq \sum_{\substack{0<d_{1}<\sqrt{D} \\
d_{1} \in \mathfrak{D}(\mathfrak{P})}} \mu^{2}\left(d_{1}\right) \prod_{p \mid d_{1}}\left(\frac{1}{\theta\left(p^{2}\right)}-1\right),
\end{aligned}
$$

where $\mu(\cdot)$ is the Möbius function. By (3.3),

$$
\frac{1}{\theta\left(p^{2}\right)}-1=\frac{2(p-1)}{p^{2}-2 p+2} \geq \frac{2}{p}
$$


hence

$$
V_{D} \geq \sum_{\substack{0<d_{1}<\sqrt{D} \\ d_{1} \in \mathfrak{D}(\mathfrak{P})}} \mu^{2}\left(d_{1}\right) \prod_{p \mid d_{1}} \frac{2}{p}=\sum_{\substack{0<d_{1}<\sqrt{D} \\ d_{1} \in \mathfrak{D}(\mathfrak{P})}} \mu^{2}\left(d_{1}\right) \frac{2^{\omega\left(d_{1}\right)}}{d_{1}}
$$

where $\omega(m)$ denotes the number of (distinct) prime divisors of $m \in \mathbb{Z}_{+}$. Let

$$
\mathfrak{P}^{\prime}:=\{p \in \mathbb{P}: p \equiv 3 \bmod 4 \text { and } p \mid k h\}, \quad \gamma(k, h):=\prod_{p \in \mathfrak{P}^{\prime}}\left(1+\frac{2}{p}\right) .
$$

Obviously,

$$
\gamma(k, h) \leq\left(\frac{k|h|}{\phi(k|h|)}\right)^{2}
$$

Furthermore, by (3.4),

$$
\begin{aligned}
\gamma(k, h) V_{D} & \geq \sum_{m_{1} \in \mathfrak{D}\left(\mathfrak{P}^{\prime}\right)} \mu^{2}\left(m_{1}\right) \frac{2^{\omega\left(m_{1}\right)}}{m_{1}} \sum_{\substack{m_{2}<\sqrt{D} \\
m_{2} \in \mathfrak{D}(\mathfrak{P})}} \mu^{2}\left(m_{2}\right) \frac{2^{\omega\left(m_{2}\right)}}{m_{2}} \\
& \geq \sum_{\substack{m<\sqrt{D} \\
m \in \mathfrak{D}\left(\mathbb{P}_{3}\right)}} \mu^{2}(m) \frac{2^{\omega(m)}}{m},
\end{aligned}
$$

where

$$
\mathbb{P}_{3}:=\{p \in \mathbb{P}: p \equiv 3 \bmod 4\}
$$

To estimate the last expression in (3.6), we consider the generating function, for $\Re(s)>1$,

$$
f(s)=\prod_{p \in \mathbb{P}_{3}}\left(1+\frac{2}{p^{s}}\right)=\sum_{m \in \mathfrak{D}\left(\mathbb{P}_{3}\right)} \mu^{2}(m) 2^{\omega(m)} m^{-s} .
$$

Evidently,

$$
f(s)=g_{7}(s) \frac{\zeta(s)^{2}}{\zeta_{\mathbb{Q}(i)}(s)}=g_{7}(s) \frac{\zeta(s)}{L(s)},
$$

where $g_{7}(s)$ is holomorphic in $\Re(s) \geq 1$, and $g_{7}(1) \neq 0$. Hence, for $s \rightarrow 1+$,

$$
f(s) \sim g_{7}(1) \frac{4}{\pi}(s-1)^{-1} .
$$

Thus Lemma D implies that

$$
\sum_{\substack{m<\sqrt{D} \\ m \in \mathfrak{D}\left(\mathbb{P}_{3}\right)}} \mu^{2}(m) \frac{2^{\omega(m)}}{m} \gg \log D
$$


Together with (3.6) and (3.5), this implies that

$$
V_{D} \gg\left(\frac{k|h|}{\phi(k|h|)}\right)^{-2} \log D
$$

Recalling (3.2) and our choice $D=\sqrt{x}$, we complete the proof of Proposition 1.

Proposition 2. For integers $k>0$ and $h \neq 0$, and large real $x$,

$$
\begin{aligned}
S_{1}(k, h ; x) & :=\sum_{0<n<x} r(n) \mathbf{b}(k n+h) \\
& \ll\left(\frac{k|h|}{\phi(k|h|)}\right)^{2} x(\log x)^{-1 / 2} .
\end{aligned}
$$

Furthermore, for each integer $h \neq 0$,

$$
S_{2}(h, x):=\sum_{0<n<x} r^{2}(n) \mathbf{b}(n+h) \ll\left(\frac{|h|}{\phi(|h|)}\right)^{2} x(\log x)^{1 / 2} .
$$

Proof. By (2.2) and a crude form of the hyperbola method,

$$
\begin{aligned}
S_{1}(k, h ; x) & \leq 4 \sum_{\substack{0<n_{1} n_{2}<x \\
n_{1}, n_{2}>0}} \mathbf{b}\left(n_{1}\right) \mathbf{b}\left(n_{2}\right) \mathbf{b}\left(k n_{1} n_{2}+h\right) \\
& \leq 8 \sum_{0<n_{1}<\sqrt{x}} \mathbf{b}\left(n_{1}\right) \sum_{0<n_{2} \leq x / n_{1}} \mathbf{b}\left(n_{2}\right) \mathbf{b}\left(k n_{1} n_{2}+h\right) \\
& =8 \sum_{0<n_{1}<\sqrt{x}} \mathbf{b}\left(n_{1}\right) S_{0}\left(k n_{1}, h ; \frac{x}{n_{1}}\right) .
\end{aligned}
$$

Hence, by Proposition 1 and (2.4) of Lemma F,

$$
\begin{aligned}
S_{1}(k, h ; x) & \ll \sum_{0<n_{1}<\sqrt{x}} \mathbf{b}\left(n_{1}\right)\left(\frac{k n_{1}|h|}{\phi\left(k n_{1}|h|\right)}\right)^{2} \frac{x}{n_{1}}\left(\log \left(\frac{x}{n_{1}}\right)\right)^{-1} \\
& \ll\left(\frac{k|h|}{\phi(k|h|)}\right)^{2} \frac{x}{\log x} \sum_{0<n_{1}<\sqrt{x}}\left(\frac{n_{1}}{\phi\left(n_{1}\right)}\right)^{2} \frac{\mathbf{b}\left(n_{1}\right)}{n_{1}} \\
& \ll\left(\frac{k|h|}{\phi(k|h|)}\right)^{2} x(\log x)^{-1 / 2}
\end{aligned}
$$

which establishes (3.7). 
Similarly, in order to show (3.8), we conclude by (2.1) that

$$
\begin{aligned}
S_{2}(h, x) & \leq \sum_{\substack{0<m k<x \\
m, k>0}} r(m) r(k) \mathbf{b}(k m+h) \\
& \leq 2 \sum_{0<k<\sqrt{x}} r(k) \sum_{0<m \leq x / k} r(m) \mathbf{b}(k m+h) \\
& =2 \sum_{0<k<\sqrt{x}} r(k) S_{1}\left(k, h ; \frac{x}{k}\right) .
\end{aligned}
$$

Therefore, by (3.7),

$$
\begin{aligned}
S_{2}(h, x) & \ll \sum_{0<k<\sqrt{x}} r(k)\left(\frac{k|h|}{\phi(k|h|)}\right)^{2} \frac{x}{k}\left(\log \left(\frac{x}{k}\right)\right)^{-1 / 2} \\
& \ll\left(\frac{|h|}{\phi(|h|)}\right)^{2} x(\log x)^{-1 / 2} \sum_{0<k<\sqrt{x}}\left(\frac{k}{\phi(k)}\right)^{2} \frac{r(k)}{k} \\
& \ll\left(\frac{|h|}{\phi(|h|)}\right)^{2} x(\log x)^{1 / 2},
\end{aligned}
$$

in view of (2.5). This completes the proof of Proposition 2.

\section{Proof of the Theorem}

Proposition 3. For $X \geq 2$ and $\frac{1}{2} X \leq x \leq X$, define

$$
H(X, x):=\frac{1}{\pi} x^{1 / 4} \sum_{1 \leq n<X^{5}} \frac{r(n)}{n^{3 / 4}} \cos \left(2 \pi \sqrt{n x}-\frac{3}{4} \pi\right),
$$

and

$$
R(X, x):=P(x)-H(X, x)
$$

Then

$$
\int_{X / 2}^{X} R(X, x)^{2} d x \ll X^{1 / 2} .
$$

Proof. By the usual asymptotics for Bessel functions,

$$
J_{1}(2 \pi \sqrt{n x})=\frac{1}{\pi}(n x)^{-1 / 4} \cos \left(2 \pi \sqrt{n x}-\frac{3}{4} \pi\right)+O\left((n x)^{-3 / 4}\right) .
$$

Using this in Lemma A, with $M=X^{5}$, the main terms of (4.3) obviously add up to $H(X, x)$. The total contribution of the $O$-terms from (4.3) to the 
left hand side of (4.2) is

$$
\ll \int_{X / 2}^{X}\left(x^{-1 / 4} \sum_{1 \leq n<X^{5}} \frac{r(n)}{n^{5 / 4}}\right)^{2} d x \ll X^{1 / 2} .
$$

Squaring and integrating the $O$-term of Lemma A, with $M=X^{5}$, we get

$$
\int_{X / 2}^{X} \min \left(X^{-4+2 \varepsilon}\|x\|^{-2}+X^{-2}, X^{2 \varepsilon}\right) d x=\int_{\substack{X / 2 \leq x \leq X \\\|x\| \leq \omega}}+\int_{\substack{X / 2 \leq x \leq X \\\|x\|>\omega}},
$$

say, where $\omega=X^{-4 / 3}$. Obviously,

$$
\int_{\substack{X / 2 \leq x \leq X \\\|x\| \leq \omega}} \ll \int_{\substack{X / 2 \leq x \leq X \\\|x\| \leq \omega}} X^{2 \varepsilon} d x \ll \omega X^{1+2 \varepsilon} \ll X^{-1 / 3+2 \varepsilon}
$$

and

$$
\int_{\substack{X / 2 \leq x \leq X \\\|x\|>\omega}} \ll\left(X^{-4+2 \varepsilon} \omega^{-2}+X^{-2}\right) X \ll X^{-1 / 3+2 \varepsilon} .
$$

Together with (4.4) this verifies Proposition 3.

Proposition 4. For positive integers $n$, let

$$
\Delta_{\mathfrak{B}}(n)=\min _{\substack{k \in \mathfrak{B} \\ k \neq n}}|k-n|,
$$

and let $H(X, x)$ be defined by (4.1). Then, for $X \geq 2$,

$$
\int_{X / 2}^{X} H(X, x)^{2} d x=C\left(X^{3 / 2}-\left(\frac{1}{2} X\right)^{3 / 2}\right)+O\left(X \sum_{1 \leq n<X^{5}} \frac{r^{2}(n)}{n \Delta_{\mathfrak{B}}(n)}\right),
$$

where $C$ is the constant given in (1.7).

Proof. For $u \geq \sqrt{X / 2}$, we put

$$
G(X, u):=\int_{\sqrt{X / 2}}^{u}\left(\sum_{1 \leq n<X^{5}} \frac{r(n)}{n^{3 / 4}} \cos \left(2 \pi \sqrt{n} t-\frac{3}{4} \pi\right)\right)^{2} d t
$$

Then, in view of (4.1),

$$
\begin{aligned}
\int_{X / 2}^{X} H(X, x)^{2} d x & =\frac{2}{\pi^{2}} \int_{\sqrt{X / 2}}^{\sqrt{X}} u^{2} \frac{\partial G}{\partial u}(X, u) d u \\
& =\frac{2}{\pi^{2}} X G(X, \sqrt{X})-\frac{4}{\pi^{2}} \int_{\sqrt{X / 2}}^{\sqrt{X}} u G(X, u) d u
\end{aligned}
$$


after a change of variable and integration by parts. We apply Lemma B to evaluate $G(X, u)$, using the identity $\cos (\alpha)=\frac{1}{2}(\exp (i \alpha)+\exp (-i \alpha))$. We choose

$$
\mathcal{J}=\left\{j \in \mathbb{Z}: 0<|j|<X^{5},|j| \in \mathfrak{B}\right\},
$$

and, for all $j \in \mathcal{J}$,

$$
a_{j}=\frac{1}{2} \frac{r(|j|)}{|j|^{3 / 4}} \exp \left(-\operatorname{sgn}(j) \frac{3}{4} \pi i\right), \quad \lambda_{j}=2 \pi \operatorname{sgn}(j) \sqrt{|j|} .
$$

For integers $n \geq 1$, write

$$
m_{\mathfrak{B}}^{>}(n):=\min \{m \in \mathfrak{B}: m>n\}, \quad m_{\mathfrak{B}}^{<}(n):=\max \{m \in \mathfrak{B}: m<n\} .
$$

Then $m_{\mathfrak{B}}^{>}(n) \asymp n \asymp m_{\mathfrak{B}}^{<}(n)$, e.g., as an immediate consequence of the classic asymptotics for $\sum \mathbf{b}(n)$. Hence, in the notation of Lemma B,

$$
\begin{aligned}
\delta_{j} & =\min _{\substack{k \in \mathcal{J} \\
k \neq j}}\left|\lambda_{k}-\lambda_{j}\right| \\
& \gg \min \left(\frac{\left|m_{\mathfrak{B}}^{>}(|j|)-\right| j||}{\sqrt{m_{\mathfrak{B}}^{>}(|j|)}+\sqrt{|j|}}, \frac{\left|m_{\mathfrak{B}}^{<}(|j|)-\right| j||}{\sqrt{m_{\mathfrak{B}}^{<}(|j|)}+\sqrt{|j|}}\right) \\
& \gg \frac{\Delta_{\mathfrak{B}}(|j|)}{\sqrt{|j|}} .
\end{aligned}
$$

Therefore, Lemma B yields

$$
\begin{aligned}
G(X, u)= & \left(u-\sqrt{\frac{1}{2} X}\right) \frac{1}{2} \sum_{1 \leq n<X^{5}} \frac{r^{2}(n)}{n^{3 / 2}}+O\left(\sum_{1 \leq n<X^{5}} \frac{r^{2}(n)}{n \Delta_{\mathfrak{B}}(n)}\right) \\
= & \frac{1}{2}\left(u-\sqrt{\frac{1}{2} X}\right) \sum_{n=1}^{\infty} \frac{r^{2}(n)}{n^{3 / 2}} \\
& +O\left(u X^{-5 / 2+\varepsilon}\right)+O\left(\sum_{1 \leq n<X^{5}} \frac{r^{2}(n)}{n \Delta_{\mathfrak{B}}(n)}\right) .
\end{aligned}
$$

Using this in (4.6), we immediately establish Proposition 4.

We are now ready to complete the proof of our Theorem. Combining Propositions 3 and 4, with an appeal to Cauchy's inequality, and summing over all intervals $\left[\frac{1}{2} X, X\right],\left[\frac{1}{4} X, \frac{1}{2} X\right], \ldots$, we obtain

$$
Q(X) \ll X \sum_{1 \leq n<X^{5}} \frac{r^{2}(n)}{n \Delta_{\mathfrak{B}}(n)} .
$$


Now, for real numbers $Z \geq 2$ and $W \geq 2$,

$$
\begin{aligned}
\sum_{\substack{\frac{1}{2} Z \leq n<Z \\
\frac{1}{2} W \leq \Delta_{\mathfrak{B}}(n)<W}} \frac{r^{2}(n)}{n \Delta_{\mathfrak{B}}(n)} & \ll \frac{1}{W Z} \sum_{\substack{\frac{1}{2} Z \leq n<Z \\
\Delta_{\mathfrak{B}}(n)<W}} r^{2}(n) \\
& \leq \frac{1}{W Z} \sum_{0<|h|<W}\left(\sum_{\frac{1}{2} Z \leq n<Z} r^{2}(n) \mathbf{b}(n+h)\right) \\
& \ll \frac{1}{W}(\log Z)^{1 / 2} \sum_{0<|h|<W}\left(\frac{|h|}{\phi(|h|)}\right)^{2} \ll(\log Z)^{1 / 2},
\end{aligned}
$$

by an appeal to (3.8). We use this estimate for $W=2,4,8, \ldots, 2^{w} \asymp \log X$, thus $w \ll \log \log X$, and

$$
\sum_{\substack{\frac{1}{2} Z \leq n<Z \\ \Delta_{\mathfrak{B}}(n) \geq 2^{w}}} \frac{r^{2}(n)}{n \Delta_{\mathfrak{B}}(n)} \ll \frac{1}{Z \log X} \sum_{\frac{1}{2} Z \leq n<Z} r^{2}(n) \ll \frac{\log Z}{\log X} \ll 1,
$$

as long as $\log Z \ll \log X$. Therefore, altogether,

$$
\sum_{\frac{1}{2} Z \leq n<Z} \frac{r^{2}(n)}{n \Delta_{\mathfrak{B}}(n)} \ll(\log X)^{1 / 2} \log \log X,
$$

if $\log Z \ll \log X$. Summing finally over $Z=X^{5}, \frac{1}{2} X^{5}, \frac{1}{4} X^{5}, \ldots$ (up to $O(\log X)$ terms) and recalling $(4.7)$, we complete the proof of our Theorem.

\section{References}

[1] T. Cochrane and R. E. Dressler, Consecutive triples of sums of two squares, Arch. Math. (Basel) 49 (1987), 301-304.

[2] K. Corrádi and I. Kátai, A comment on K. S. Gangadharan's paper "Two classical lattice point problems", Magyar Tud. Akad. Mat. Fiz. Oszt. Közl. 17 (1967), 89-97 (in Hungarian, with English summary).

[3] H. Cramér, Über zwei Sätze des Herrn G. H. Hardy, Math. Z. 15 (1922), 201-210.

[4] J. L. Hafner, New omega theorems for two classical lattice point problems, Invent. Math. 63 (1981), 181-186.

[5] E. Hlawka, J. Schoißengeier, und R. Taschner, Geometrische und analytische Zahlentheorie, Manz, Wien, 1986.

[6] M. Huxley, Exponential sums and lattice points II, Proc. London Math. Soc. (3) 66 (1993), 279-301.

[7] —, Exponential sums and lattice points III, ibid. 87 (2003), 591-609.

[8] A. Ivić, The Laplace transform of the square in the circle and divisor problems, Studia Sci. Math. Hungar. 32 (1996), 181-205.

[9] I. Kátai, The number of lattice points in a circle, Ann. Univ. Sci. Budapest. Eötvös Sect. Math. 8 (1965), 39-60 (in Russian). 
[10] E. Krätzel, Lattice Points, Kluwer, Dordrecht, 1988.

[11] - Analytische Funktionen in der Zahlentheorie, Teubner, Stuttgart, 2000.

[12] E. Landau, Über die Gitterpunkte in einem Kreise, Vierte Mitteilung, Nachr. Königl. Ges. Wiss. Göttingen Math.-Phys. Kl. 1923, 58-65.

[13] Y.-K. Lau and K.-M. Tsang, Mean square of the remainder term in the Dirichlet divisor problem, J. Théor. Nombres Bordeaux 7 (1995), 75-92.

[14] T. Meurman, On the mean square of the Riemann zeta-function, Quart. J. Math. Oxford Ser. (2) 38 (1987), 337-343.

[15] H. L. Montgomery and R. C. Vaughan, Hilbert's inequality, J. London Math. Soc. (2) 8 (1974), 73-82.

[16] Y. Motohashi, Lectures on Sieve Methods and Prime Number Theory, Tata Institute of Fund. Research, Bombay, 1983.

[17] E. Preissmann, Sur la moyenne quadratique du terme de reste du problème du cercle, C. R. Acad. Sci. Paris Sér. I Math. 306 (1988), 151-154.

[18] G. J. Rieger, Aufeinanderfolgende Zahlen als Summen von zwei Quadraten, Indag. Math. 27 (1965), 208-220.

[19] A. Selberg, Remarks on multiplicative functions, in: Number Theory Day (New York, 1976), Lecture Notes in Math. 626, Springer, Berlin, 1977, 232-241.

[20] K. Soundararajan, Omega results for the divisor and circle problems, Int. Math. Res. Not. 2003, no. 36, 1987-1998.

[21] A. Walfisz, Teilerprobleme, Math. Z. 26 (1927), 66-88.

Institut für Mathematik

Department für Integrative Biologie

Universität für Bodenkultur Wien

Peter Jordan-Straße 82

A-1190 Wien, Austria

E-mail: nowak@mail.boku.ac.at

http://www.boku.ac.at/math/nth.html

Received on 22.7.2003

and in revised form on 10.10.2003 